\title{
Iatrogenic bile duct injuries during laparoscopic cholecystectomy - Early management and the role of endoscopy and surgery
}

\author{
Krishnakumar Madhavan, MBBS, MS, FRCSEd, FRCS
}

Associate Professor, Department of Surgery, National University Health System, Singapore

\begin{abstract}
Key Words: Bile duct injuries, Endoscopic retrograde cholangiopancreatography
\end{abstract}

Early management of a bile duct injury after laparoscopic cholecystectomy begins with an early diagnosis. The injuries that are diagnosed at the time of surgery will usually result in an immediate conversion to open surgery and a primary repair by an experienced hepatobiliary surgeon. More commonly, a duct injury is suspected when a patient after a difficult cholecystectomy complains of an undue amount of pain (often delaying discharge) or bilious fluid is noted in a drain. Other times, a patient who was initially discharged gets readmitted in the early postoperative period with abdominal pain, fever or jaundice. The surgeon should have a very high index of suspicion under any of these circumstances as early detection and intervention are mandatory for a satisfactory outcome. Abdominal examination may not contribute much unless the patient has peritonitis and some form of imaging study will be necessary. Blood tests may show derangement of liver functions or a high leucocyte count. An ultrasound examination may reveal either free intraperitoneal fluid or intrahepatic biliary dilatation, but usually a contrast enhanced CT scan is necessary for more detailed information (including possible injury to the vascular structures). A detailed MRCP will often demonstrate the breach in bile duct and the site of leak but often the clinician resorts to an ERCP to demonstrate these and also to therapeutically intervene at the same time if appropriate.

Let us consider the approach to a suspected bile duct injury under different circumstances. If bile is coming out of a post-operative drain in the immediate postCorrespondence: Krishnakumar Madhavan, Associate Professor, Department of Surgery, National University Health System, Singapore

Email: krishnakumar_madhavan@nuhs.edu.sg

The Sri Lanka Journal of Surgery 2011; 29(2):87-88 operative period, it is unlikely that a tubogram ( screeing after instillation of a contrast dye into the drain) will be of much help. One option is to take the patient back to the operating theatre, reintroduce a laparoscope, wash out the bile and look for a source of leak. The only situation it can help is an inadvertent division of a duct of Luschka in the gall bladder bed and if this can be clearly demonstrated, this can be controlled laparoscopically by clipping or suturing such a small duct. If a drain was not used initially and the patient is being investigated for increasing abdominal pain or peritonism, a relaparoscopy can help the surgeon to place 1-2 drains in the subhepatic region as a prelude to sending the patient to a specialist centre for further management. If the surgeon himself is experienced in biliary surgery or has colleagues in the hospital who are biliary surgeons, it may be advisable to take his/her help during this relaparoscopy and even prepare the patient for a laparotomy if indicated after an initial laparoscopy. Another option under these circumstances is to get an early ERCP and if this demonstrates a "minor" injury either in the form of a slipped clip on the cystic duct or indeed a leak from a divided duct of Luschka, a temporary stent in the bile duct may resolve the problem.

On the other hand, if a patient who has been discharged after surgery is readmitted with symptoms suspicious of a biliary injury, prompt imaging either in the form of a CECT or MRCP or both should be performed. If the exact nature of the injury is not obvious after these investigations, an ERCP may be helpful both for diagnostic and therapeutic purposes. The usual presentation will be with jaundice or persistently deranged liver functions or attacks of cholangitis. Very rarely some patients may present after many years with secondary biliary cirrhosis. In a patient who presents late, thorough pre-operative assessment 
should be done and surgery if indicated, carefully planned. In a small proportion of patients, major liver resection with or without biliary reconstruction or indeed a liver transplantation may be the only option .In late strictures, surgical options are superior to endoscopic ones. Hence endoscopic stenting is chosen for patients who are either unfit for surgery or unwilling to undergo surgery.

In this context it will be important to mention the debate regarding "early" surgical repair in iatrogenic bile duct injuries. Much has been written on this, but now there is a general consensus that if the opportunity arises for an early repair (no intra-abdominal sepsis, available surgical expertise, detection within the first week to 10 days) a primary repair done by a specialist biliary surgeon is safe and gives very good results both in long and short term. Any form of major liver resection should be avoided during this early repair but may be the only option in associated vascular injuries.

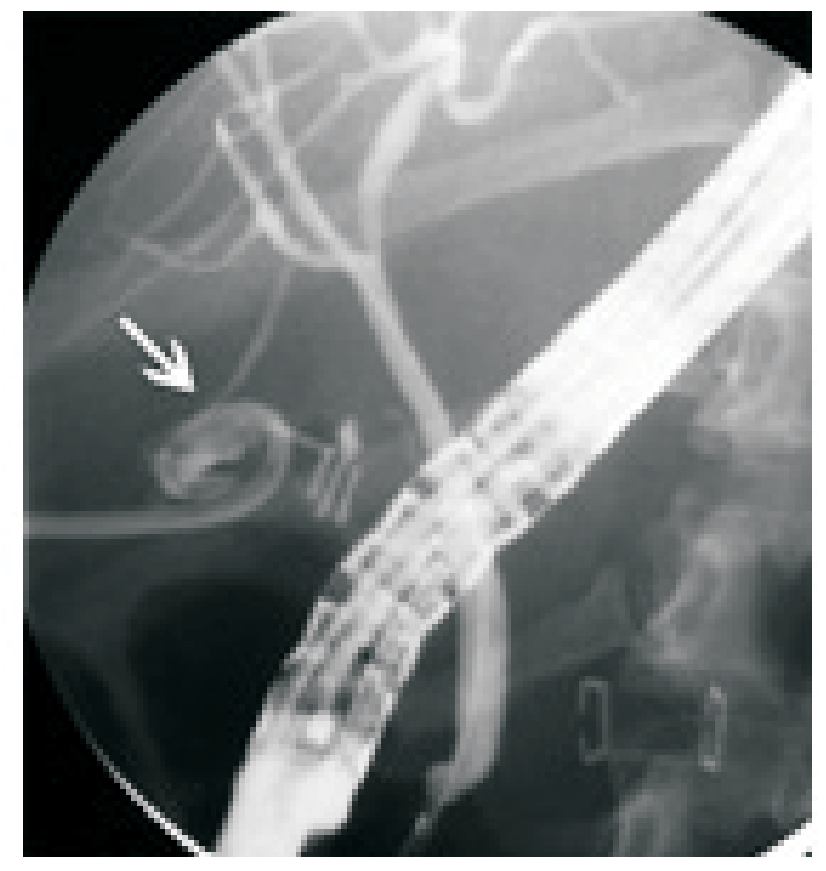

Figure 1. A bile leak from a disrupted duct of Luschka following laparoscopic cholecystectomy demonstrated at ERCP. This was successfully treated with a biliary stent and a percutaneous drainage of the bile collection (Courtesy Google Images)

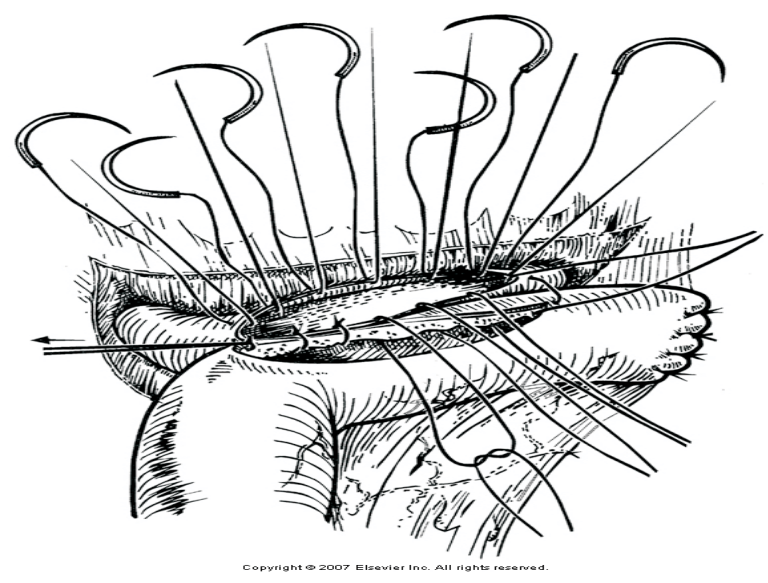

Figure 2. A high left duct hepaticojejunostomy (the Hepp Couinaud approach) the preferred surgical approach to a bile duct injury where the left and right hepatic ducts are in communication (Courtesy Surgery of the Liver, Biliary tract and pancreas , Fourth Edition -Saunders Elsevier)

\section{References}

1. Savader SJ, Lillemo KD, Prescott CA, Lund GB et al. Laparoscopic cholecystectomy related bile duct injuries: a health and financial disaster.Ann Surg 1997;225:268273.

2. Stewart L, Way LW. Bile duct injuries during laparoscopic cholecystectomy . Factors that influence the results of treatment. Arch Surg 1995; 130:1123 1129.

3. Thomson BNJ, Parks RW, Madhavan KK, Wigmore SJ and Garden OJ. Early specialist repair of biliary injury. British Journal of Surgery 2006; 93:216-220.

4. Nordin A, Halme L, Makisalo $\mathrm{H}$ et al. Management and outcome of major bile duct injuries after laparoscopic cholecystectomy : from therapeutic endoscopy to liver transplantation . Liver Transpl .2002; 8(11):1036-43.

5. Thomson BNJ, Parks RW, Madhavan KK and Garden OJ. Liver resection and transplantation in the management of iatrogenic biliary injury. World J Surg 2007; 31:2363-9. 